\title{
基于晶格的人工内分泌系统模型及在多机器人 系统中的应用
}

\author{
王䂞 ${ }^{(1)}$, 徐庆征 ${ }^{(1) *}$ \\ (1) 西安理工大学计算机科学与工程学院, 西安 710048 \\ (2) 西安通信学院, 西安 710106 \\ * 通信作者. E-mail: xuqingzheng@hotmail.com
}

收稿日期: 2010-04-19; 接受日期: 2010-07-22

国家自然科学基金 (批准号: 60802056. 61073091)、陕西省自然科学基金 (批准号: 2010JM8028) 和西安大学优秀博士学位论文 研究基金 (批准号: 105-211010) 资助项目

\begin{abstract}
摘要 为了解决多机器人系统分布式控制问题, 借鉴人体内分泌系统研究的理论成果, 提出基于晶 格的人工内分泌系统模型. 该模型以环境晶格化为基础, 以细胞智能化为依托, 以累加激素为纽带, 以靶细胞为导向, 能够适应外环境的持续变化, 保持内环境的相对稳定, 充分体现人体内分泌系统的 自组织和自修复特性. 实验结果表明, 无需复杂的集中控制策略, 基于该人工内分泌系统模型的多机 器人系统能够在未知或危险环境中执行搜索和捕获多目标的任务.
\end{abstract}

\section{关键词人工内分泌系统 多机器人系统 内分泌细胞 激素自组织自修复 搜索和捕获}

\section{1 引言}

基于生物体信息处理机制的生物智能方法研究是人工智能领域的一个热点, 也是现代科学发展过 程中多学科、多领域间相互交叉和深度融合的必然结果和集中体现. 生物体内存在三大调节系统 脑神经系统、免疫系统和内分泌系统, 它们之间相互交织和渗透, 构成一个立体的智能调节网络, 通过 细胞因子、神经递质和激素进行双向信息传递和协同作用, 共同负责机体对持续变化的内环境和外环 境的适应性反应, 维持机体器官和组织的长期平衡状态, 使生物体具备代谢、生长、生育、生殖、思维、 运动等功能. 在现代脑神经科学和认知科学的研究成果的基础上, 人们提出了人工神经网络 (artificial neural network, ANN), 它模仿和延伸人脑智能、思维、意识等认知功能, 显示出很强的自适应性、鲁 棒性、自组织、自学习和容错能力, 具有广泛的应用领域 ${ }^{[1-3]}$. 自然免疫系统是一种高度复杂的分布 式并行处理系统, 具有很强的学习、记忆和联想能力, 对检测和消除感染问题显示出精确的调节能力. 近一二十年以来, 基于固有免疫系统、适应免疫系统、克隆选择理论、免疫网络理论、危险理论等机制 和理论的人工免疫系统 (artificial immune system, AIS) 研究也取得了丰硕的研究成果, 展现出旺盛的 生命力 ${ }^{[4-6]}$. 人工神经网络和人工免疫系统的理论、模型与应用所取得的巨大成功, 充分显示出生物 智能方法研究的重大理论意义和实用价值, 同时也激发了人们开展对包括内分泌系统在内的其他生物 信息处理系统进行研究的兴趣和热情. 相比较而言, 人工内分泌系统 (artificial endocrine system, AES) 
的研究方兴未艾, 尚处于学科创立和起步阶段, 并且进展缓慢, 存在许多亟待解决的理论盲区和工程 应用方面的技术难点.

1984 年, Mori 提出的自治分布系统 (autonomous decentralized systems, ADS) 可能是最早使用基 于激素的方法来建立鲁棒、灵活、在线修复系统的工作 ${ }^{[7,8]}$. 在 ADS 中, 采用基于消息内容的编码通 信协议来通信, 随后, ADS 系统应用于其他的工业问题中, 表现出良好的在线扩展、在线保持、错误容 忍特性 [9].

激素计算模型是一类具有明显生物学背景的 AES 理论模型. 美国哈佛医学院 Kravitz 教授以龙虾 作为研究对象, 系统阐述了激素、神经激素与神经系统相互配合, 共同控制龙虾行为的原理 (图 1) ${ }^{[10]}$. Brooks 以该行为控制模型为基础, 提出了激素计算模型 (computational model of hormones) ${ }^{[11]}$, 它采 用激励水平来描述行为被激励的程度. 基于激素计算模型构建的机器人展现出良好的生存能力和高效 性, 而且行动选择过程中不会产生冲突现象, 但是在学习能力和伸缩性等方面尚待提高和完善. 随后, Avila-Garcia 提出激素调节模型 ${ }^{[12,13]}$, 在行为决策过程中充分发挥情感的作用, 是一个理想的处理内 部和外部刺激的解决方案.

以 Turing 的反应一扩散模型为基础, Shen 将随机推理和行动、动态网络配置、分布式控制、自 组织、自适应和自学习机制有机融合在一起, 提出数字激素模型 (digital hormone model, DHM) ${ }^{[14-16]}$. 该模型包括 3 个部分: 动态网络、单个机器人行动选择的概率函数、激素反应、扩散和消耗的数学方 程. 数字激素模型具有结构简单、数学定义清晰、应用领域广泛等优点 ${ }^{[17-19]}$, 很有希望成为一个通 用的 AES 理论模型, 但该模型中机器人缺乏协商和合作机制, 因此难以处理动态目标和多目标等复杂 状况.

机器人学方向或许是最早开展人工内分泌系统应用研究, 也是最成功的领域之一. Shen 提出基于 生物激素概念的分布式控制机制 ${ }^{[20-24]}$, 基本思想是将自重构系统看作是一个网络, 网络中的节点就 是单个自治机器人, 拥有独立的能量、流程、加速器、传感器和连接器, 节点间通过传送激素信息来联 系和交流. 将激素看作是触发不同模块不同行为的信号, 行为一旦被触发就由各个模块独立执行选定 的任务. Mendao 利用激素信号来协调单个自治机器人同时完成多个任务 ${ }^{[25]}$. 每一个任务对应一个腺 体, 并以固定的速率释放激素到激素池中, 当激素池中的激素满足一定数量要求, 它就释放出来变成自 由激素, 开始在全局范围内传播. 其中, 具有最高浓度的自由激素驱动行为控制模块执行相应的任务, 而且在执行任务期间, 该腺体停止释放激素. 随后, Walker 将其扩展到多个自治机器人的任务分配系 统 ${ }^{[26]}$, 在没有中央控制的前提下以较低的复杂度动态地分配任务, 对于各个机器人和外部环境的改变 能够主动地做出正确的反应. 机器人之间广播目前执行的任务, 当某个机器人收到该信息后, 它停止释 放相关的激素, 激素池中的激素数量也要相应衰减. 然而, 该系统对于微小的环境改变和参数调整过 于敏感, 直接影响系统整体性能.

人工内分泌系统还应用于人机情感交流 ${ }^{[27-29]}$ 、多处理器智能控制 [30-32]、解耦控制器 ${ }^{[33-35] 、}$

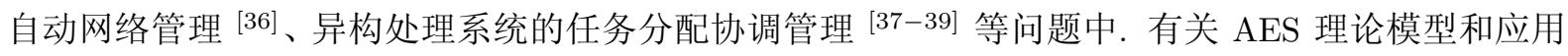
研究的现状, 请参见文献 [40]

伴随着生物内分泌科学研究成果的日益丰富和完善, 以及人工内分泌系统理论研究的逐渐深入和 应用领域的不断拓展, 人们逐渐意识到现有的理论模型和应用算法所固有的一些缺陷. 首先, 人体内 激素的种类众多, 目前已知的就有 200 多种, 它们来源复杂, 生理作用各异, 广泛分布于血液、组织液、 细胞间液、细胞内液或神经节囊泡空隙等部位. 在上述模型和算法中, 内分泌细胞生理结构和作用完 全相同, 难以体现出激素结构的多样性以及激素间相互作用形式的复杂性. 其次, 激素的有效浓度是 


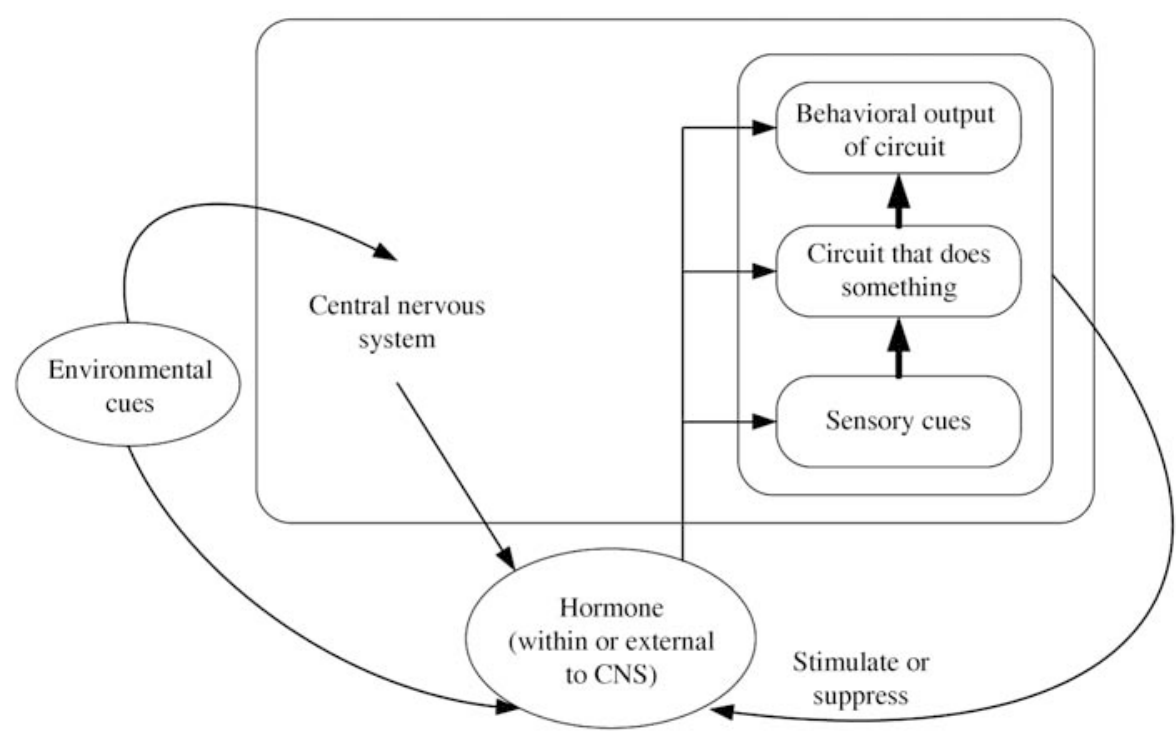

图 1 龙虾的行为控制原理 ${ }^{[10]}$

Figure 1 Diagrammatic representation of hormonal sensitization of the response of sets of neurons ${ }^{[10]}$

由激素合成与释放速率、降解与转换速率共同决定的, 所有这些过程受到脑神经系统的精细调节, 使 得激素的生理浓度维持在合理的范围内. 大多数模型和算法均假定激素的降解与转换等代谢过程瞬间 完成, 而且代谢得十分彻底, 因此, 当前的激素浓度仅仅与当前的内分泌细胞分布有关, 而与历史分布 无关. 最后, 以上理论模型和应用算法往往强调和模拟生物内分泌系统的某些局部特征, 难以全面揭 示内分泌系统的整体特性, 例如自组织和自修复性. 针对上述问题, 借鉴生物内分泌系统信息处理机 制, 特别是受激素间协同作用的启发, 本文提出基于晶格的人工内分泌系统 (lattice-based AES, LAES) 模型.

\section{2 内分泌系统概述}

人们对内分泌现象的认识虽可追溯到很久远的年代, 但内分泌学作为一门独立的学科则不过百年 的历史. 近年来, 由于分子生物学、细胞生物学、免疫学、遗传学、生态学、心理学和临床医学等学科 新理论、新技术的不断渗透和影响, 现代内分泌学各领域都发生了革命性的变化和发展 ${ }^{[41-43]}$, 人们 对内分泌系统的信息处理机制也有了更加深入和全面的理解.

\section{1 内分泌系统的组成}

内分泌系统是机体重要的生理调节系统, 由内分泌腺体和散布于全身不同组织的分泌细胞所组成, 依靠激素传递信息, 在细胞的生长、分化、调亡及保持机体内环境稳定中发挥着重要作用. 按照经典 的定义, 激素是指由生物体的内分泌细胞分泌, 经血液循环系统运送到其他远处组织器官并调节靶细 胞功能的高效生物活性物质.

蛋白质、肽类激素的合成经历转录、翻译和翻译后等过程. 受到内分泌细胞刺激后, 囊泡与细胞 膜融合, 贮存在囊泡中的激素就从内分泌细胞中释放出来. 胺类和类固醇激素均以酪氨酸和胆固醇为 
原料, 通过一系列的酶促反应, 转变成各种不同的类固醇激素. 由于类固醇激素是高度脂溶的, 它们在 细胞内合成后可通过简单的扩散作用出胞, 直接进入血液循环系统.

激素的释放具有阶段性, 也就是说, 大多数激素的释放是在短时间内突然发生的, 在两次突发释 放之间很少或不释放激素. 因此, 血浆中激素的浓度会在短时间内迅速波动.

激素运输的路线有长有短, 形式多样. 在血液循环过程中, 一部分激素与特定的血浆蛋白结合, 而 且不同的激素与血浆蛋白结合的程度有很大差别, 另一部分激素以游离状态在血液中运输. 需要特别 指出的是, 结合型激素无活性, 它必须转变为游离型激素才具有相应的生理作用.

激素从释放出来到消失所经历的生命周期有长有短, 一般采用半衰期来衡量激素的有效期. 肽类 激素的半衰期较短,一般为 3-7 min. 类固醇类激素的半衰期随激素的类型和分子结构而异, 但一般较 肽类激素长, 多数为数小时, 少数可长达数周以上.

\section{2 激素间的作用方式}

现代内分泌医学研究发现, 各种内分泌腺虽位于身体不同部位, 但其分泌的激素的作用并不孤立, 而是相互联系相互影响的, 共同形成一个完整的精巧的调节基本生命过程的内分泌系统, 使之具有很 强的可变性、精确的等级性和较高的安全系数. 普遍认为, 激素间存在以下几种相互作用的形式:

(1) 一种激素多重作用. 许多激素在不同组织中或同一组织的不同生命周期中, 表现出不同的生 理作用. 例如, 睪酮具有促进男性胚胎的性分化, 刺激男性泌尿生殖道生长, 调节精子生成和促红细胞 生成素的合成, 促进性毛和体毛的发育与生长, 促进前列腺增生等功能. 从基因及现代分子生物学观 点来看, 一种激素的多重作用具有相同的作用机制.

(2) 多种激素同一功能, 即激素的协同作用. 在人体内, 几乎所有的生理功能、病理反应等生命过 程都是在多种激素和多种细胞因子的共同控制和相互作用下完成的. 例如升血糖这一生命过程是在胰 高糖素、肾上腺素、正肾上腺素、皮质醇、生长激素的直接作用下, 以及甲状腺激素影响食欲、生长抑 素抑制胰岛素和胰高糖素的释放及抑胃肽增强胰岛素分泌等间接作用下产生的.

(3) 激素的相反作用, 即呈拮抗作用. 如甲状旁腺具有生血钙的作用, 相反, 降钻素具有降血钻的 作用.

(4) 允许作用. 激素本身不能在所作用的器官或细胞上直接引起某种生理效应, 但它的存在能使 其他组织对某些生理信息 (神经冲动、激素或代谢产物) 的敏感性增加, 是其他激素产生生理效应的必 要条件. 例如, 去甲肾上腺素的升压作用必须依赖于皮质醇的存在.

当然, 激素间还存在一种所谓的中性作用, 即两种激素之间彼此不受影响.

\section{LAES 模型}

LAES 模型的基本思想是在晶格网络这个环境空间内, 内分泌细胞群体动态分布和移动, 无需集 中控制策略或 “领导者” 细胞, 各内分泌细胞仅利用累加激素信息相互通信, 协调各自动作, 在靶细胞 的干预和指导下, 完成特定的任务. 累加激素信息没有地址标识, 在整个晶格网络内部自由传播. 每个 内分泌细胞依据所获取的累加激素信息、局部拓扑结构和自身状态信息来决定下一步如何行动.

从数学的角度考虑, LAES 可以抽象为一个五元组模型 $\mathrm{LAES}=\left(L_{d}, \mathrm{EC}, \mathrm{TC}, H, A\right)$. 式中, LAES 代表一个基于晶格的人工内分泌系统, 它由环境空间 $L_{d}$ 、内分泌细胞 EC、靶细胞 $\mathrm{TC} 、$ 激素 $H$ 及算 法 $A$ 等 5 部分共同组成 ${ }^{[44]}$. 下一小节着重介绍前 4 部分, 3.2 小节给出算法 $A$ 的详细描述. 


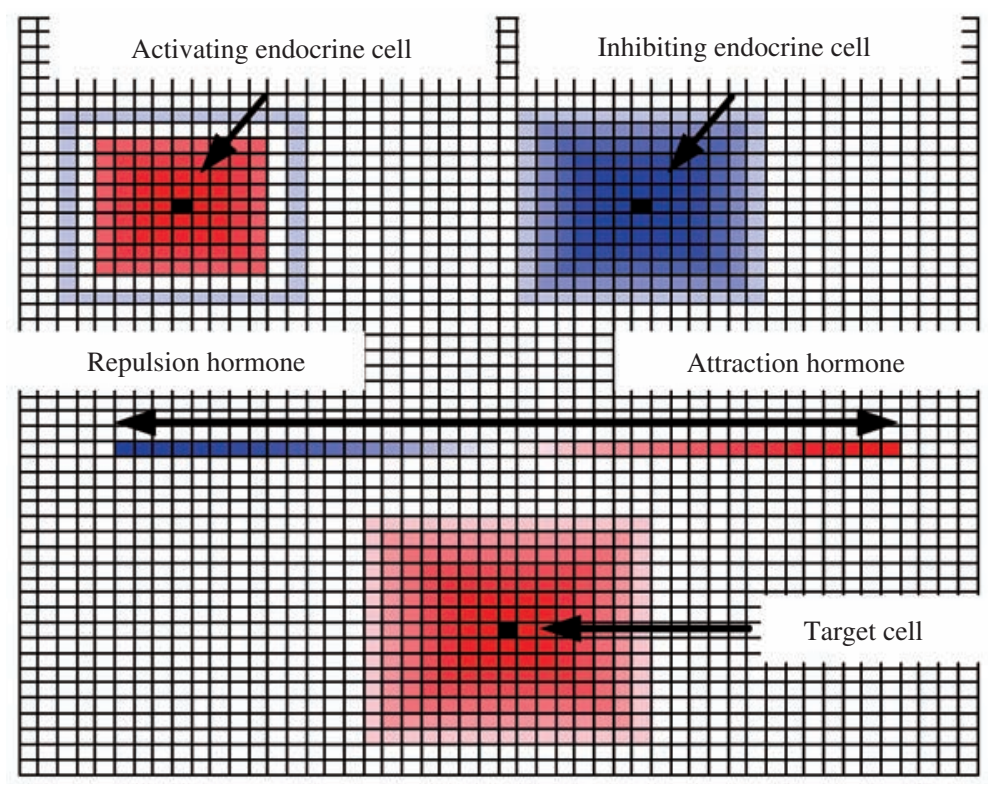

图 2 基于晶格的人工内分泌系统

Figure 2 Lattice-based artificial endocrine system

\subsection{LAES 模型设计}

在我们的模型中, 所有的组成要素只能在有限的范围内生存、通信、移动或消亡, 我们称该有限 的范围为环境空间 $L_{d}$, 其中 $d$ 是正整数, 表示环境空间的维数. 我们首先研究 $d=2$ 的情形, 在时机成 熟时, 再将相关研究思路、方法和成果推广到高维情形. 人体内分泌系统在十分广阔的范围内发挥重 要的调节作用, 内分泌细胞自由地分布在整个环境中, 并占据一定的空间位置. 为处理方便且不失一 般性, 我们首先将二维的环境空间离散化, 分解为多个晶格单元, 显然, 离散化的尺度越小, 晶格的数 目越多. 此处, 晶格的形状没有特别的限制, 通常为标准的正方格 (如图 2 背景所示), $L_{x y}$ 表示位于第 $x$ 行第 $y$ 列的晶格单元所处的状态, 其中 $L_{x y}=0$ 表示该晶格单元处于空闲状态, $L_{x y}=-1$ 表示该晶 格单元为障碍物. 而且, 每个晶格内只能存在一个内分泌细胞.

内分泌细胞 EC 又可称为单元或基本单元, 是人工内分泌系统中最基本的组成部分. 出于降低成 本和复杂性的考量, 单个内分泌细胞必须尽可能的简单. 本文中, 每个内分泌细胞由负责感知相邻晶 格内激素浓度的传感器 $A_{E}$ 和激素释放器 $B_{E}$ 构成. 如前所述, 激素释放的过程具有阶段性, 持续时 间十分短暂. 据此, 我们将内分泌细胞释放激素的过程离散化, 释放器 $B_{E}$ 仅在相应的时刻释放一定 量浓度的激素. 另外, 内分泌细胞种类繁多, 生理功能不尽相同, 我们根据晶格单元内相邻两个时刻激 素浓度变化情况, 将内分泌细胞分为刺激型内分泌细胞 $\mathrm{EC}_{A}$ 和抑制型内分泌细胞 $\mathrm{EC}_{I}$ 两类. $L_{x y}=1$ 表示该晶格单元的激素浓度升高了, 此时为刺激型内分泌细胞 (如图 2 左上部所示); $L_{x y}=2$ 表示该 晶格单元的激素浓度降低了, 此时为抑制型内分泌细胞 (如图 2 右上部所示).

靶细胞 TC 是指能够接受内分泌细胞分泌激素刺激的器官或细胞, 它具有与激素特异性结合的受 体 (如图 2 下部所示). 通常, 靶细胞就是需要完成的任务或者需要达成的若干个目标, 它可以抽象为 一个激素释放器 $B_{T}$, 并持续不断的释放一定量浓度的激素, 以吸引周围的内分泌细胞. 需要特别注意 的是, 内分泌细胞不会凭空产生, 也不会消亡, 只能按规则在环境空间内自由移动. 当内分泌细胞移动 到靶细胞所处的位置, 并完成既定任务后, 靶细胞自动消亡, 并停止释放激素. 
激素是由内分泌腺体或内分泌细胞分泌的高效生物活性物质, 通过调节各组织细胞的代谢活动影 响人体的生理活动. 本文中, 激素 $H$ 包括排斥激素 $H_{r}$ 和吸引激素 $H_{a}$ 两类 (如图 2 中部所示). 通常, 靶细胞只释放吸引激素, 以吸引周围的内分泌细胞, 并指导其完成特定的任务. 抑制型内分泌细胞只释 放排斥激素, 以阻止其他内分泌细胞重复搜索相同或相近的区域. 刺激型内分泌细胞既释放排斥激素, 又释放吸引激素, 且吸引激素的浓度大于排斥激素的浓度, 其目的在于促使更多的内分泌细胞沿着相 近的道路搜索, 增加捕获靶细胞的概率.

激素的作用范围是有限的, 该作用范围内的空间域叫做邻居. 原则上, 对邻居的大小和形状没有 限制, 参考元胞自动机模型中邻居的选取方式, 本文采用扩展的 Moore 型邻居 ${ }^{[45]}$ 作为激素的作用范 围, 表示为

$$
N_{\text {Moore-r }}=\left\{\left(N_{x}, N_{y}\right)|| N_{x}-x|\leqslant r,| N_{y}-y \mid \leqslant r,\left(N_{x}, N_{y}\right) \in \mathbb{Z}^{2}\right\},
$$

其中, $N_{x}, N_{y}$ 表示邻居细胞的行、列坐标值, $x, y$ 表示中心细胞的行、列坐标值, $r$ 表示邻居半径.

在我们的模型中, 激素释放以后, 通过血液循环系统在相应的邻居内转运, 运输路线的长短由邻 居半径 $r$ 决定. 在运输过程中, 激素以结合态和游离态两种形式存在, 且仅有游离态激素具有生理活 性. 我们假定, 处于不同位置的游离态激素的浓度大体遵循正态分布, $t$ 时刻 $L_{a b}$ 处的内分泌细胞 $j$ 释 放的第 $i$ 类激素转运到 $L_{x y}$ 处游离态激素的浓度, 描述如下:

$$
H_{i j}(x, y, t)=\frac{a_{i}}{2 \pi \sigma_{i}^{2}} \mathrm{e}^{\frac{(x-a)^{2}+(y-b)^{2}}{2 \sigma_{i}^{2}}}, \quad|x-a| \leqslant r, \quad|y-b| \leqslant r,
$$

其中, $\sigma_{i}^{2}$ 为标准方差, 反映转运过程中损耗的快慢, $a_{i}$ 为常数, 反映游离态激素的比重.

随着时间的推移, 在转换或降解等代谢过程的共同作用下, 激素浓度会逐渐降低, 甚至消耗殆尽, 需要内分泌细胞合成和释放新的激素, 以维持机体激素浓度的自然平衡. $L_{x y}$ 处的的累加激素浓度 $H(x, y, t)$ 由经过代谢消耗后, 上一时刻遗留的激素 $H^{\prime}(x, y, t)$ 和当前时刻多个内分泌细胞合成和释 放的激素 $H^{i j}(x, y, t)$ 共同构成, 描述如下:

$$
\begin{aligned}
H(x, y, t) & = \begin{cases}\sum_{j=1}^{m} \sum_{i=1}^{n} H_{i j}(x, y, t)+H^{\prime}(x, y, t), & t>1, \\
\sum_{j=1}^{m} \sum_{i=1}^{n} H_{i j}(x, y, t), & t=1,\end{cases} \\
H^{\prime}(x, y, t) & =(1-\alpha) H(x, y, t-1),
\end{aligned}
$$

其中, $m$ 表示邻居内内分泌细胞的数量, $n$ 表示激素种类, $\alpha$ 为代谢消耗系数.

\section{2 算法设计}

算法 A 表示人工内分泌系统各要素相互协作, 共同完成特定任务的流程, 伪代码如图 3 所示.

在 LAES 模型中, 内分泌细胞的移动规则 $R$ 是指, 根据内分泌细胞状态信息、累加激素信息以及 局部拓扑结构确定下一时刻该内分泌细胞移动方向的动力学函数. 显然, 所有的内分泌细胞遵守相同 的移动规则, 尽管移动规则仅依赖于局部信息, 但它极大地影响着系统的整体性能, 使之表现出复杂的 行为状态.

假定内分泌细胞所处晶格的累加激素浓度为 $h_{0}$, 它周围紧邻的 8 个晶格的累加激素浓度分别为 $h_{1}, h_{2}, h_{3}, \ldots, h_{8}$, 如图 4 所示. 基于上述定义, 移动规则 $R$ 描述如下: 


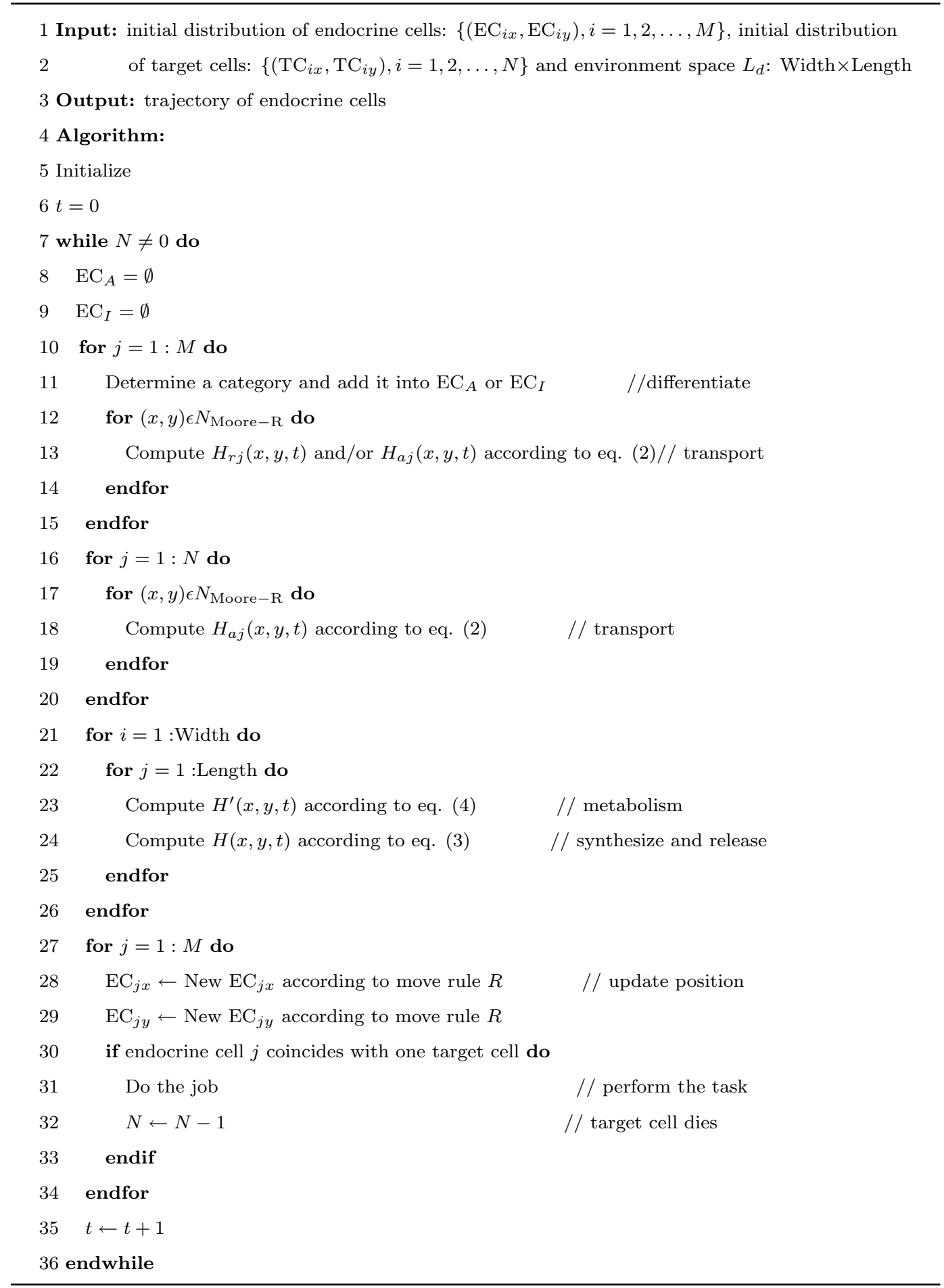

图 3 算法 $\mathbf{A}$ 的伪代码

Figure 3 Pseudocode for algorithm A

步骤 1 根据累加激素的浓度计算各个晶格的选择概率 $p_{i}(i=0,1,2, \ldots, 8)$. 本文采用的方法可 表示如下: 


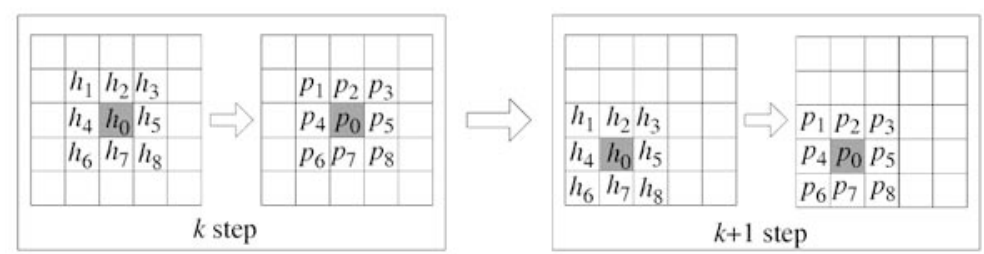

图 4 内分泌细胞依据规则移动的实例

Figure 4 Example of an endocrine cell moves according to the move rule

$$
p_{i}= \begin{cases}10 \times h_{i}, & h_{i}>0 \\ 1, & h_{i}=0 \\ -\frac{1}{h_{i}}, & h_{i}<0\end{cases}
$$

步骤 2 内分泌细胞依据轮盘奢方式确定下一步移动的方向.

步骤 3 各个内分泌细胞首先虚拟移动. 如果多个内分泌细胞占据同一晶格, 则随机移动到距离 最近的空闲晶格.

本文采用的移动规则 $R$ 既保证内分泌细胞以较大概率选择吸引激素浓度较高的晶格单元, 又简 化计算过程, 提高算法效率.

\section{3ＬAES 模型的自组织和自修复特性}

内分泌系统虽然也受神经系统的影响和控制, 但其主要功能都是通过内部各个子部分的相互作用 来自组织实现的. 内分泌系统是一个高度一体化的系统, 生命体内的各种激素形成一个连锁的复合体, 它们从不单独发生作用, 一种激素的作用受到其他激素的调整或制约, 某一腺体功能过剩或不足可改 变另一腺体产生激素的速率. 有大量的例子表明, 一些激素和另一些激素的作用可产生相反的效应. 例 如, 胰岛素有全面降低血糖的作用, 而胰高血糖素的作用正好相反. 某些垂体前叶细胞受正中隆起双重 促垂体激素控制, 一种促进分泌物的释放, 另一种抑制其释放. 总之, 内分泌系统通过内部各个子系统 相互间的局部协同作用来突现出其整体的动态平衡特性.

先天发育或者后天疾病可以导致腺体机能的光进或低下, 表现为某种激素的过多或缺乏, 从而在 生命体征上显现为病态或亚健康状态, 甚至出现内分泌系统疾病. 现代分子内分泌学研究表明, 生命 机体能够采取有效措施, 维持激素水平的平衡, 预防内分泌疾病的发生. 通常情况下, 同一生理功能由 多种激素来完成, 如果缺乏某种激素, 可以合成、转化和释放较多的其他激素来代替该激素, 维持生理 功能的稳定.

本文的目的之一就是检验 LAES 模型是否具有类似的自组织和自修复特性. 为此, 我们试图回答 和讨论如下几个问题:

(1) LAES 模型是否可以借助简单的移动规则, 自组织地形成某种模式?

(2) 内分泌细胞的种群规模对最终形成的模式的影响如何?

(3) 内分泌细胞释放激素的数量和分布对最终形成的模式的影响如何?

(4) 通常情况下, 两类内分泌细胞数量的变化趋势如何?

(5) 遇到外部环境发生剧烈变化时, LAES 模型能否维持某种模式? 


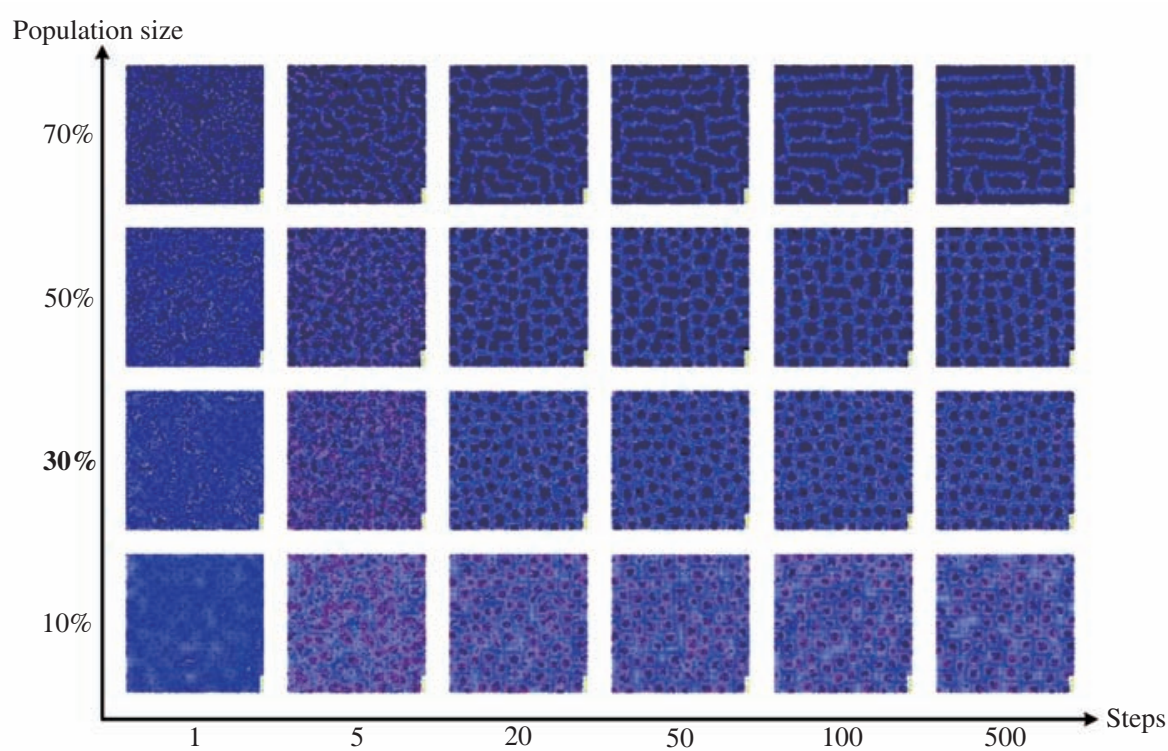

图 5 种群规模的影响

Figure 5 Pattern formation with different cell population sizes

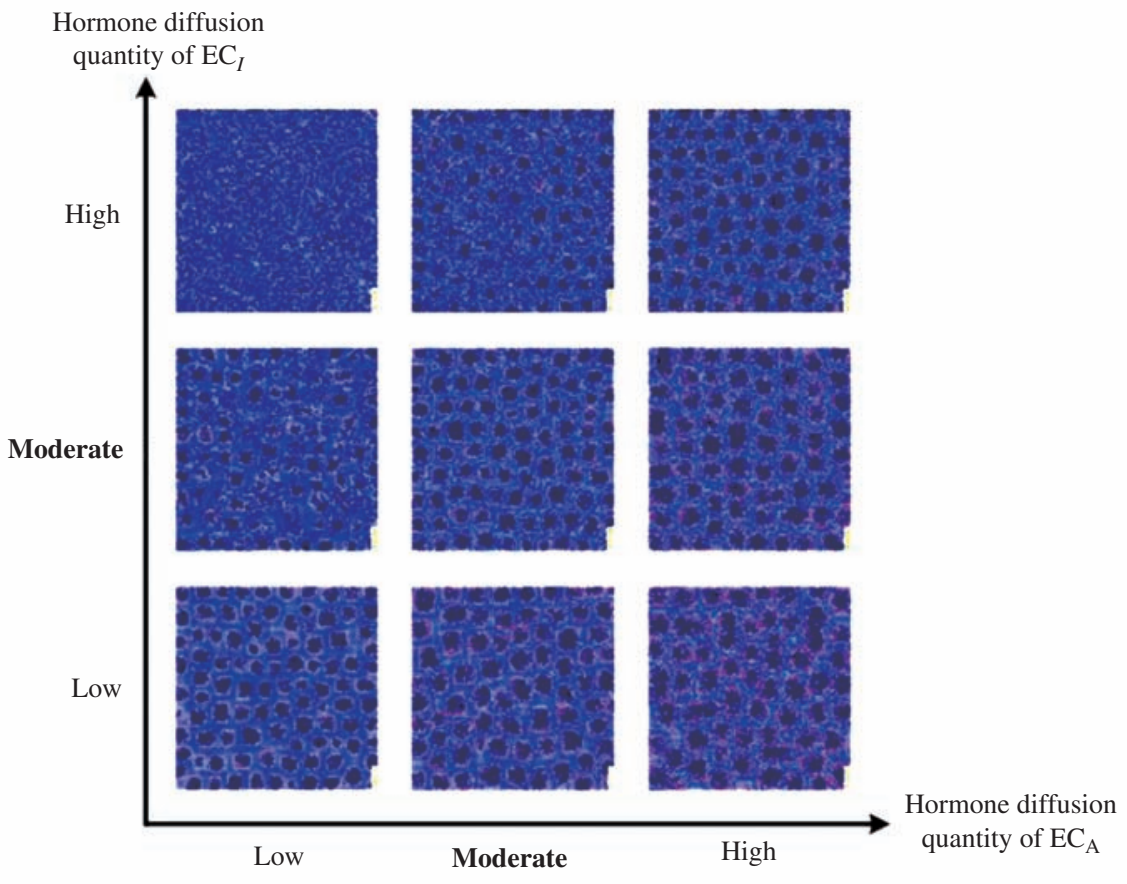

图 6 释放激素的数量的影响

Figure 6 Pattern formation with different quantities of hormone diffusion

为了回答上述问题, 我们设计了相应的 4 组实验. LAES 模型采用 JAVA 语言来实现, 实验数据 采用 SPSS 14.0 来处理和分析, 运行环境为 Pentium IV $2.4 \mathrm{GHz}$, 内存为 $512 \mathrm{MB}$, 人工内分泌系统的 环境空间为 $100 \times 100$ 的二维晶格网络. 
第 1 组实验中, 内分泌细胞释放激素的数量和分布是相同的, 内分泌细胞种群的规模从 $10 \%$ (约 1000 个内分泌细胞) 到 $70 \%$ (约 7000 个内分泌细胞) 之间变化. 最初这些细胞在整个环境空间内是随 机分布的, 每次实验执行 500 步, 分别记录第 1, 5, 20, 50, 100, 500 步时内分泌细胞和激素浓度的分布 情况, 结果如图 5 所示. 所有的实验结果都表明, 内分泌细胞形成了一定的模式. 当种群规模相对较小 时, 内分泌细胞形成许多相对孤立的聚类 (如图 5 下面 2 行), 而且, 聚类的大小依赖于细胞的种群规 模. 相反, 如果细胞种群规模不断增大, 这些相对孤立的聚类在大量吸引激素的作用下相互靠拢, 内分 泌细胞逐步形成带状模式 (如图 5 上面 2 行). 需要注意的是, 带状模式的方向可以是水平的也可以是 垂直的, 且具有不可预测性. 即使对于相同的初始分布, 由于单个内分泌细胞行为的不确定性, 内分泌 系统所展现出的带状模式的方向, 既可能是水平的也可能是垂直的.

第 2 组实验中, 内分泌细胞的种群规模和内分泌细胞释放激素的分布是相同的, 内分泌细胞释放 激素的数量是变化的, 大致分为多、适中、少 3 种情况. 当两类内分泌细胞释放激素的数量比较平衡 时 (如图 6 右上 - 左下对角线所示), 经过 500 步的迭代, 内分泌细胞种群形成的模式清晰可见. 当刺 激型内分泌细胞释放激素数量占优时 (如图 6 右下部分所示), 聚类规模在逐渐增大, 而且优势越明显, 聚类规模也越大. 相反, 当抑制型内分泌细胞释放激素数量占优时 (如图 6 左上部分所示), 人工内分 泌系统几乎不能形成某种模式. 当 $\mathrm{EC}_{I}$ 释放激素数量远远多于 $\mathrm{EC}_{A}$ 释放激素的数量时, 即使仿真时 间无限延长, 内分泌细胞也不会形成任何模式. 这就说明并不是所有激素数量都能使得人工内分泌系 统表现出自组织特性.

第 3 组实验中, 内分泌细胞的种群规模和内分泌细胞释放激素的数量是相同的, 我们希望观察到 内分泌细胞释放激素分布的变化对于最终形成模式的影响. 从图 7 中可以看出, 内分泌细胞释放激素 的分布处于相对平衡状态时 (如图 7 第 3 行所示), 内分泌细胞形成的模式和第 1 组实验的结果是一 致的. 而且, 聚类规模会随着吸引激素的增多而逐渐变大. 当吸引激素数量达到最大时, 或者说, 仅存 在吸引激素时, 内分泌细胞形成的聚类规模也最大 (如图 7 第 4 行所示). 相反, 如果排斥激素的数量 累积到一定程度, 即使仿真时间无限延长, 内分泌细胞也不会形成任何模式 (如图 7 第 1 行所示). 这 也说明并不是所有的激素分布情况都能使得人工内分泌系统表现出自组织特性.

选取上述 3 组实验中的相同部分 (图 5-7 中粗体部分), 作为 LAES 模型的典型实验设置. 为了消 除内分泌细胞初始分布以及模型自身迭代引起的随机误差, 独立运行典型实验 20 次, 观察两类内分泌 细胞数量的变化趋势. 从图 8 中可以看出, 最初, 人工内分泌系统的晶格内不存在浓度变化情况, 我们 将所有内分泌细胞都认为是抑制型内分泌细胞. 第 2 代时, 大量的细胞分化为刺激型内分泌细胞, 随 后, 刺激型内分泌细胞数量逐渐减少, 抑制型内分泌细胞数量逐渐增加. 在第 20 代左右, 两者数量不 再发生明显变化, 趋于一种动态平衡. 大量仿真实验表明, 数量变化曲线的峰值、趋于稳定的快慢以及 两类内分泌细胞的数量比例受到多种因素的影响, 但是两类细胞数量的变化趋势大体相同. 需要说明 的是, 即便最初内分泌细胞全部是刺激型内分泌细胞, 我们也能得到相似的结果.

实验 4 的目的是观察 LAES 模型对于意外损害的自修复特性. 首先假定人工内分泌系统已经形 成了模式, 趋于相对稳定状态 (如图 9 第 1 列所示). 由于某种因素的影响, 导致人工内分泌系统中心 部分大约 $15 \%$ 的内分泌细胞遭到破坏 (如图 9 第 2 列所示). 我们观察到, 经过若干次迭代, 人工内分 泌系统能够再次形成某种模式, 趋于另一个稳定状态. 这就说明, 内分泌细胞按照简单的行为规则行 事, 在没有全局信息的指导下, LAES 模型仍然能够形成新的模式, 体现出生物内分泌系统的自修复特 性. 而且, 修复的速度也比较快, 大约为 500-1000 代左右. 


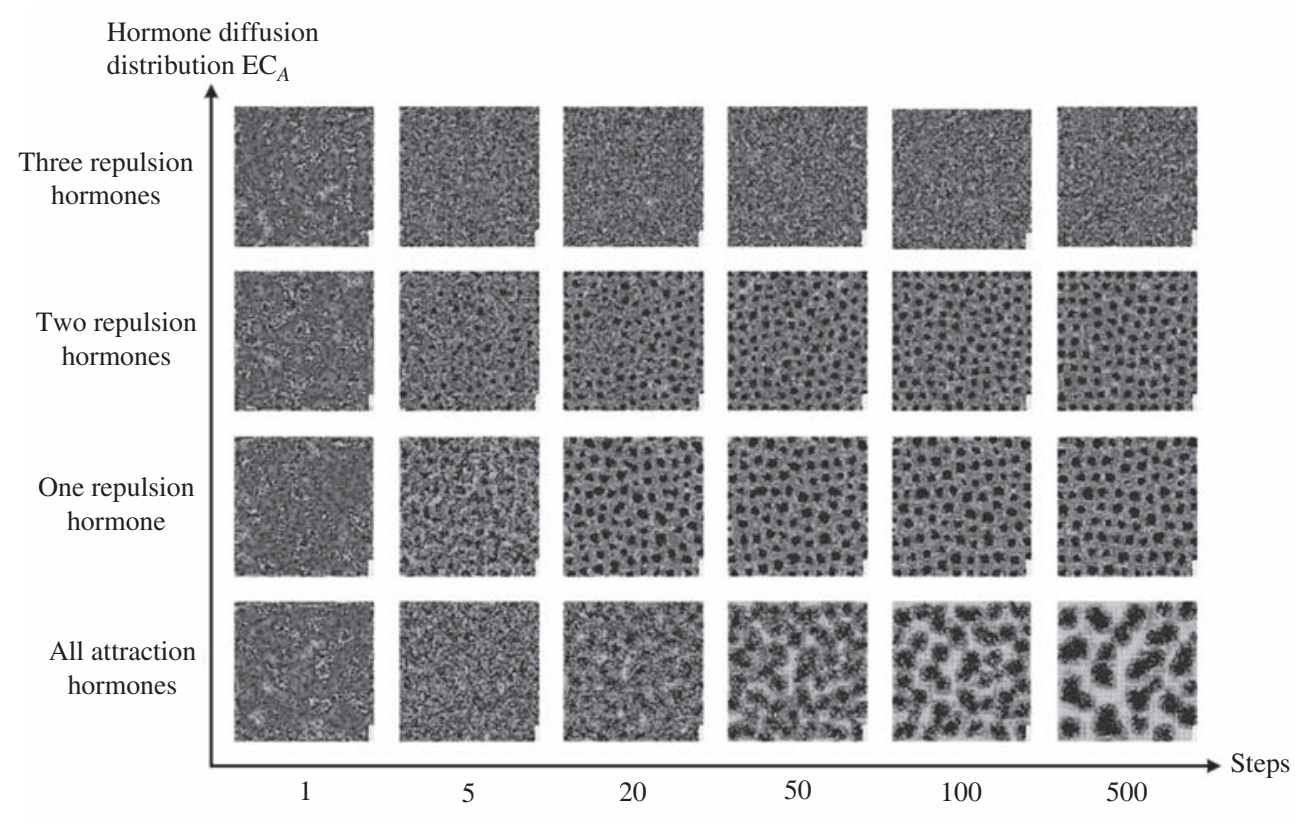

图 7 释放激素的分布的影响

Figure 7 Pattern formation with different hormone diffusion distribution

\section{LAES 模型在多机器人系统中的应用}

伴随着大规模集成电路、嵌入式计算、低能耗无线通信技术的快速发展, 以及工业、农业、国防等 领域的迫切需求, 多机器人系统的研究已经成为机器人学研究的一个重要方向. 多机器人系统具有许 多单机器人系统所没有的优点, 例如空间上的分布性、功能上的多样性、操作时的并行性以及更高的 可靠性和更低的经济成本等. 为了使多机器人系统具有适应复杂的未知环境的能力, 机器人必须在与 外部环境和其他机器人的相互作用中, 不断地学习和进化其协作行为, 这是多机器人搜索和捕获研究 中最有潜力的研究内容之一, 将在地道、敌营等危险环境探测以及火灾、地震等自然灾害搜救活动中 得到日益广泛的应用. LAES 模型表现出良好的自组织和自修复特性, 不仅较好的模拟了人体内分泌 系统的基本特征, 而且与多机器人系统的基本要求不谋而合. 尽管生物内分泌系统和多机器人系统之 间在智能化程度、通信类型等方面存在诸多差异, 但两者在系统架构、功能特点、实现方式等方面仍 存在一一对应的关系, 如表 1 所示.

\section{1 应用于多机器人系统的算法设计}

基于以上分析, 我们将 LAES 模型中的基本算法 A 扩展为可应用于多机器人系统的算法, 为叙述 方便, 称之为算法 A-robotic, 其伪代码如图 10 所示. 与基本算法 A 相比较, 新算法 A-robotic 引入了 增强型内分泌细胞 $\mathrm{EC}_{E}$ 和中性内分泌细胞 $\mathrm{EC}_{N}$ 两个概念. 其中, 增强型内分泌细胞是从内分泌细胞 种群中随机分离出来的, 它释放一定量的吸引激素, 有利于其他机器人沿着相似的路径前进, 但它采 取随机移动的方式来搜索全局空间, 不受相邻激素浓度的影响, 体现了人体激素间的允许作用. 内分 泌细胞一旦进入靶细胞的作用区域, 就转化为中性内分泌细胞, 这种内分泌细胞不释放任何种类的激 


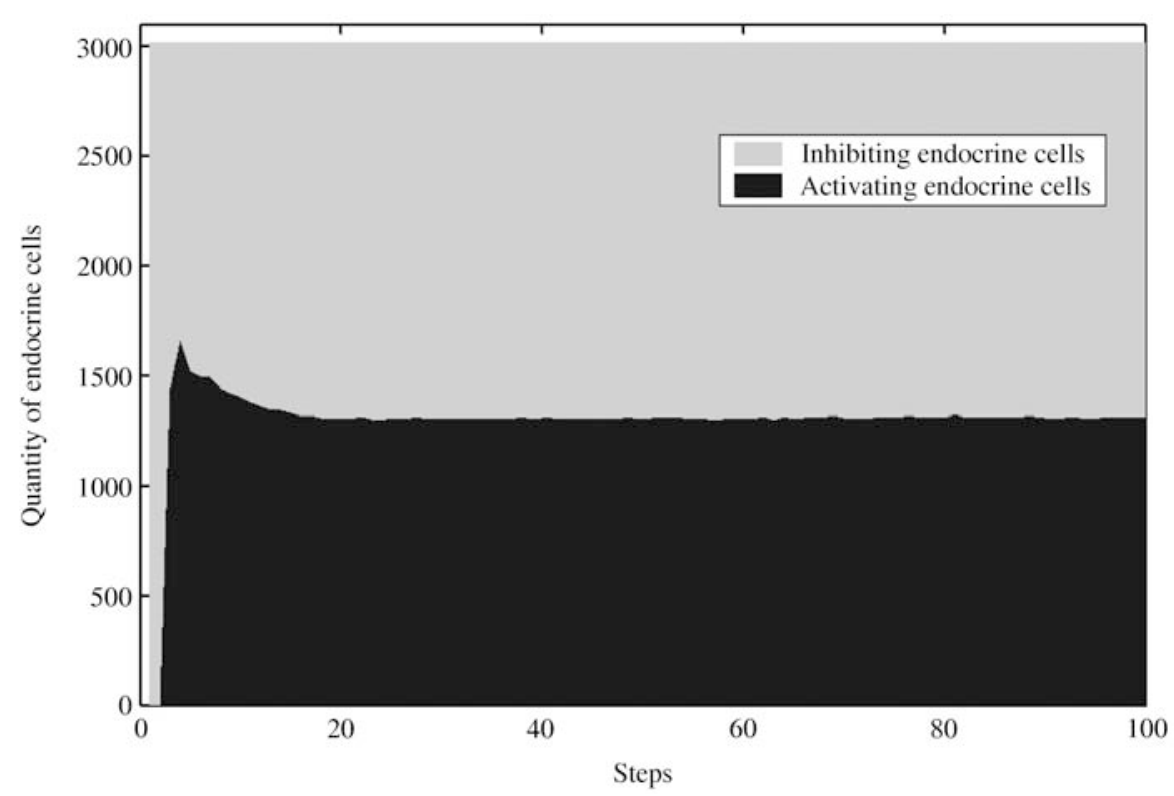

图 8 两类内分泌细胞的数量变化

Figure 8 Variation in quantity of two kinds of endocrine cells

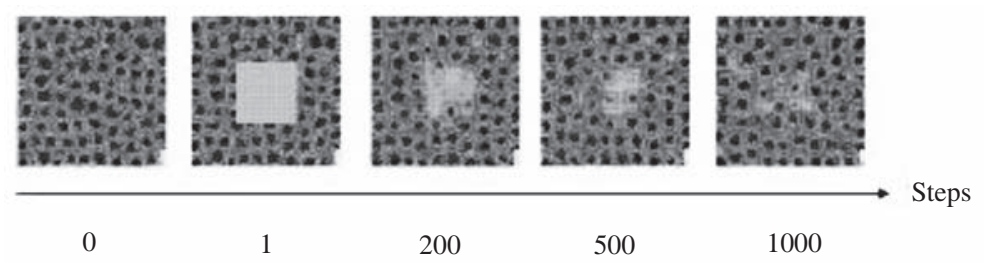

图 9 LAES 模型的自修复特性

Figure 9 Self-repairing feature of LAES model

\section{表 1 生物内分泌系统与多机器人系统的对应关系}

Table 1 Correspondence between the endocrine system and robotic swarms

\begin{tabular}{ll}
\hline Endocrine system & Robotic swarms \\
\hline Living body & Environment space/Motion domain \\
Endocrine cell & Robot \\
Endocrine cell population & A swarm of robots \\
Hormone & Short range wireless signal (either RF or infrared) \\
Hormone releaser $B_{E}$ and $B_{T}$ & Signal transmitter \\
Hormone preceptor $A_{E}$ & Signal receiver \\
Target cell & Target for seizing \\
Blood circulatory system & Free space \\
Barriers such as other cells & Barriers such as walls and rivers \\
\hline
\end{tabular}

素, 因而不会对其他机器人的行为活动产生影响, 另一方面, 它也不受其他机器人的影响. 这就体现了 人体内分泌系统中激素间所谓的中性作用. 


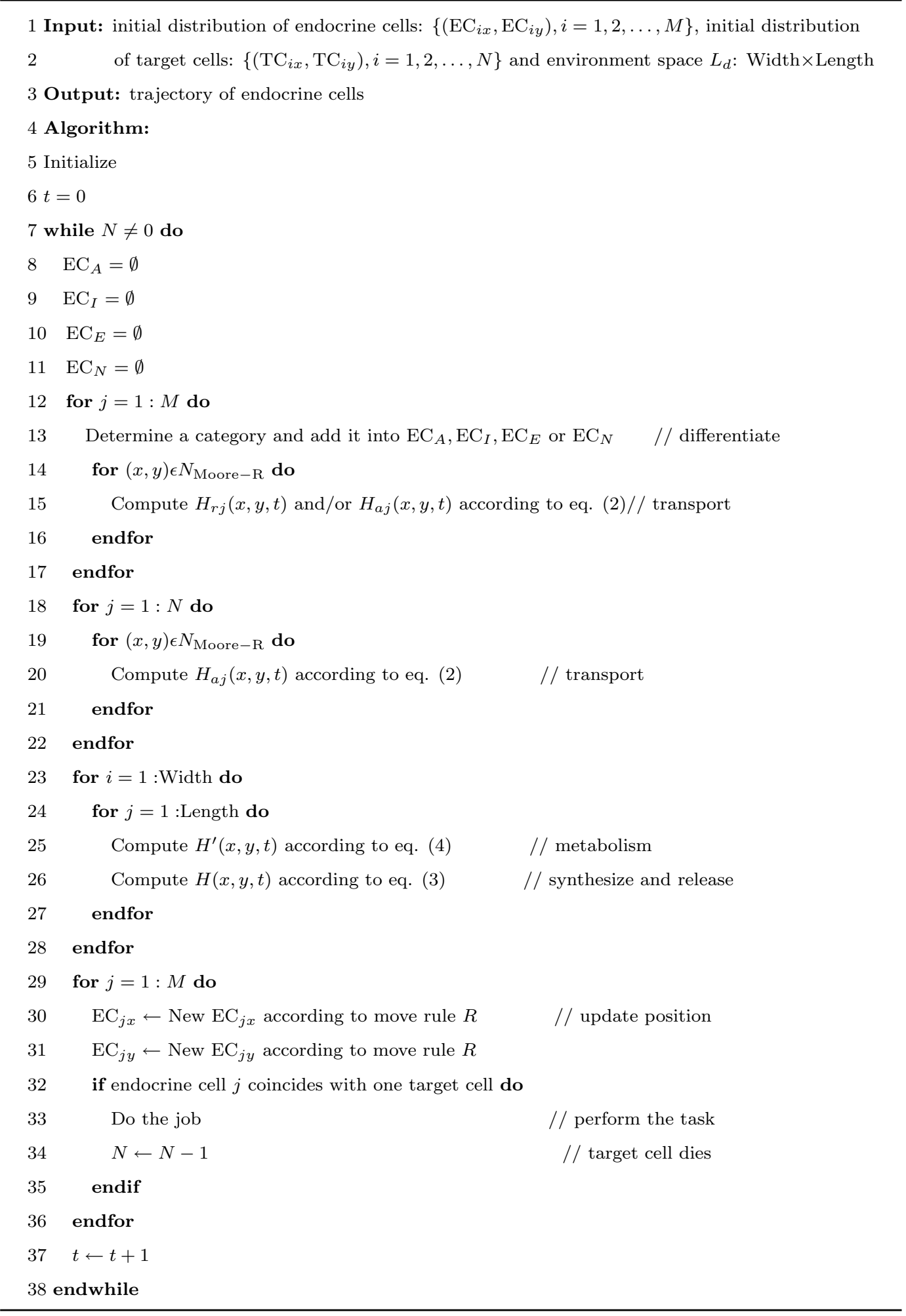

图 10 算法 A-robotic 的伪代码

Figure 10 Pseudocode for the A-robotic algorithm 


\section{2 实验结果}

第 1 组实验的目的是检验基于 LAES 模型的多机器人系统在未知环境下的搜索能力 (图 11). 场 景设置相对简单, 整个环境空间被墙体等障碍物分割为 3 个区域, 每个墙体有两扇门来连接各个相互 独立区域. 我们假定无线信号可以穿越墙体. 起初, 所有的机器人都位于左部区域. 在激素信息的作用 下, 一些机器人进入到中部区域, 随后又进入到右部区域. 这就表明, 无需复杂的集中控制策略, 基于 LAES 模型的机器人可以在任意环境下展开有效的搜索活动, 这对探测危险环境时减少人员损失会产 生积极作用. 图 12 显示了 20 次独立实验不同区域内分泌细胞数量变化的平均值, 从中也可以看出, 随着时间的不断延续, 中部区域和右部区域内机器人分布数量在持续增加.

在第 2 组实验中, 我们假定在环境空间中存在两个靶细胞, 分别位于 $(60,10)$ 和 $(60,50)$ 晶格处, 它们可以被机器人感知和识别, 多机器人系统的任务就是搜索和捕获这些靶细胞 (图 13). 起初, 所有 的内分泌细胞位于左上角, 集中分布在 $(8,8)$ 附近. 机器人首先在全局空间中搜索, 很快, 一些机器人 会被靶细胞释放的激素吸引. 最终, 机器人可以捕获到该靶细胞. 值得注意的是, 并非所有的机器人都 搜索和捕获同一个靶细胞, 存在大量的机器人在一个更加开放的环境空间搜索其他潜在目标. 搜索和 捕获目标两种任务分配的平衡性, 一方面是由于 LAES 模型不是基于确定性函数的, 而是基于概率函 数的, 另一方面是由于引入增强型内分泌细胞的缘故, 它使得多机器人系统在全局搜索目标和局部捕 获目标之间处于一种动态平衡状态.

在搜索和捕获目标的过程中, 机器人不可避免地会遇到一些障碍物, 例如房屋、墙体、沟壑、河流 等. 第 3 组实验主要是检验基于 LAES 模型的多机器人系统绕过障碍物的能力 (图 14). 我们假定内 分泌细胞同样集中分布在 $(8,8)$ 附近, 环境空间中存在一个靶细胞, 位于 $(85,85)$ 晶格处, 设置一组障 碍物, 在 $(35,35)$ 处呈十字状分布. 从图 14 中可以看出, 当越来越多的机器人集中于障碍物附近时, 会 释放大量的抑制激素, 使得一些机器人可以从侧面绕过障碍物. 反过来, 这些自由的机器人又可以吸引 其他机器人绕过障碍物. 随着越来越多机器人选择正确的搜索路径, 机器人系统最终可以捕获到靶细 胞. 通常情况下, 障碍物的属性和分布是任意复杂且难以预料的. 但是, 借助 LAES 模型的自组织和自 修复特性, 基于 LAES 模型的多机器人系统有能力克服障碍物, 并成功捕获目标.

表 2 统计了 20 次独立实验各性能指标的平均值和均方差. 人工内分泌系统的环境中存在障碍物 以后, LAES 模型经过若干的迭代, 能成功越过障碍物, 并继续搜索和捕获靶细胞, 但迭代次数和花费 时间等性能指标有一定程度的降低. 有意思的是, 我们的障碍物设置比较特别, 留有两个出口, 右上方 出口宽度为 15 , 左下方出口宽度为 5 . 我们统计了算法停止时, 内分泌细胞位于环境空间不同区域的 数量分布情况. 由表 2 可以看出, 右上部分的内分泌细胞数量与左下部分内分泌细胞数量之比大约为 1.5 , 显然不是 $\frac{15}{5}=3$. 原因在于随着时间的不断推移, 绕过障碍物的内分泌细胞会在全部的自由空间 有规则的移动, 起初分布在不同区域的内分泌细胞会逐渐融合.

\section{5 结论}

人体内分泌系统表现出很强的信息处理能力, 也为许多工程应用问题带来重要启示, 人工内分泌 系统的研究与应用正体现了这一发展趋势. 受内分泌系统信息处理机制的启发, 本文提出基于晶格的 人工内分泌系统模型. 该模型允许大量小型简易内分泌细胞相互通信, 协同作用, 构成一个大规模系 统. 即使处于一个急剧变化的、不可预测的环境中, 也不需要内分泌细胞的地址标识, 通过细胞间的协 同配合就可以形成并维持某种全局模式, 体现出人体内分泌系统的自组织和自修复特性. 这非常适合 


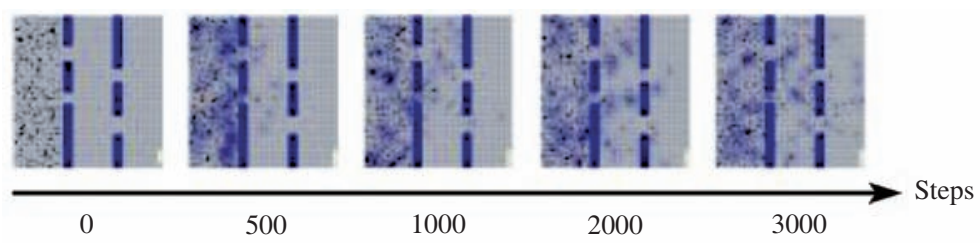

图 11 搜索未知环境

Figure 11 Example of searching unfamiliar environments

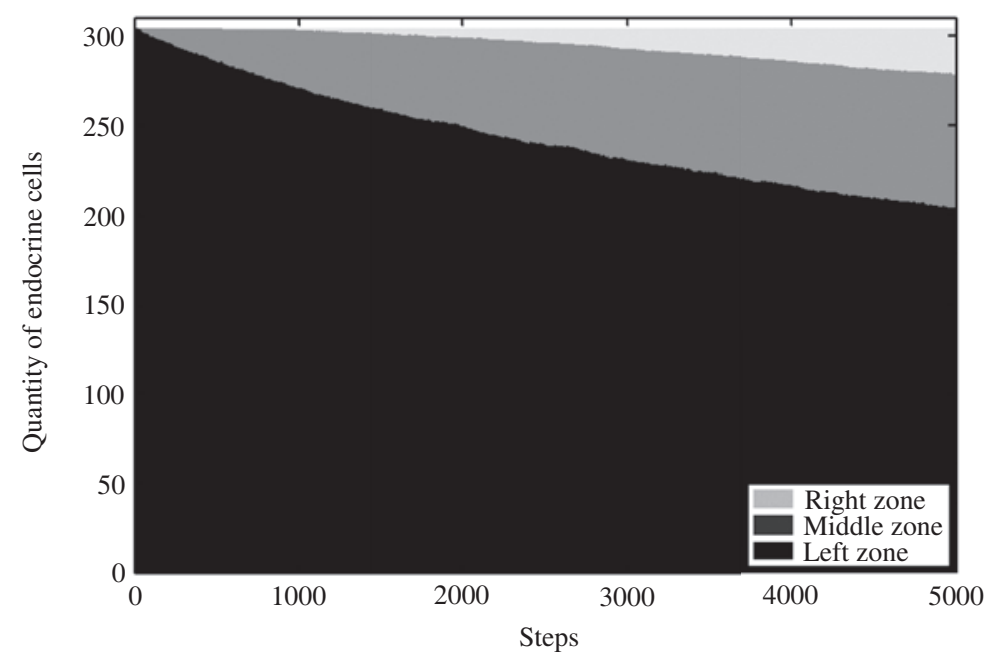

图 12 不同区域内分泌细胞数量

Figure 12 Number of endocrine cells in three zones
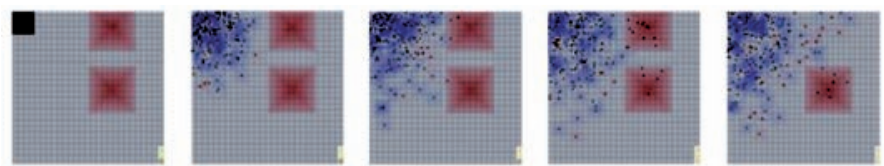

0

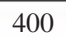

605

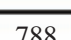

图 13 搜索和捕获多目标

Figure 13 Example of searching and seizing multiple targets

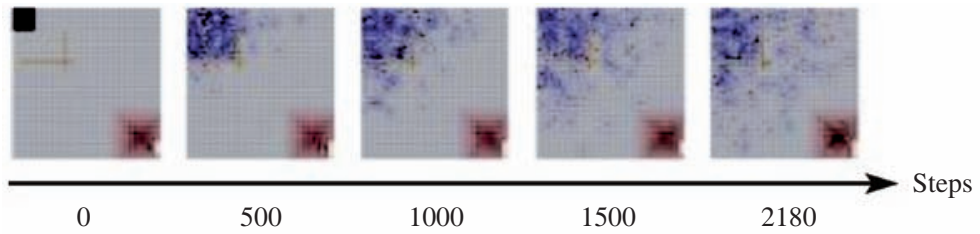

图 14 绕过障碍物

Figure 14 Example of bypassing the barrier and seizing a target 
表 2 不同场景下多机器人系统性能对比

Table 2 Comparison of robotic swarms system in different senarios

\begin{tabular}{ccccccc}
\hline \multirow{2}{*}{ Barrier } & Steps & Running time $(\mathrm{s})$ & & \multicolumn{3}{c}{ Number of free robots } \\
\cline { 4 - 6 } & & & Upper right & Bottom left & Middle & Total \\
\hline No & $1721 \pm 252$ & $118 \pm 19$ & $70 \pm 8$ & $70 \pm 6$ & $2.1 \pm 1.2$ & $142 \pm 10$ \\
Yes & $2286 \pm 442$ & $144 \pm 40$ & $65 \pm 8$ & $43 \pm 8$ & $1.5 \pm 0.7$ & $110 \pm 14$ \\
\hline
\end{tabular}

于在缺乏有效集中控制策略的前提下, 多机器人系统在复杂未知环境中搜索和捕获多目标的现实问 题. 大量的仿真实验表明, LAES 模型具有简单性、鲁棒性和自组织性等优良特性, 是模拟和分析内分 泌系统自组织和自修复现象的有力工具, 同时, 它为开发和测试新假设、新理论提供了一个坚实的实 验基础.

下一步研究工作是, 着重考察 LAES 模型在动态环境或连续系统中的表现和性能. 例如: 障碍物 或靶细胞处于快速移动过程、算法执行以后内分泌细胞或靶细胞数量增加或减少, 甚至某个靶细胞需 要多个内分泌细胞的协同工作等. 此外, 与其他经典的智能计算方法进行性能比较也是一项有意义的 工作.

致谢 感谢 University of Southern California 的 Wei-min Shen 博士提供热心帮助.

\section{参考文献}

1 Hassoun M H. Fundamentals of Artificial Neural Networks. Cambridge: MIT Press, 1995. 35-56

2 Rabunal J R, Dorrado J. Artificial Neural Networks in Real-life Applications. Hershey: Idea Group Publishing, 2005. 47-346

3 Graupe D. Principles of Artificial Neural Networks. 2nd ed. New Jersey: World Scientific Publishing Company, 2007. $1-16$

4 Dasgupta D. Artificial Immune Systems and Their Applications. Heidelberg: Springer, 1998. 157-290

5 De Castro L N, Timmis J. Artificial Immune Systems: a New Computational Intelligence Approach. Heidelberg: Springer, 2002. 269-318

6 Dasgupta D, Nino F. Immunological Computation: Theory and Applications. Florida: Auerbach Publications, 2008. $171-204$

7 Ihara H, Mori K. Autonomous decentralized computer control systems. Computer, 1984, 17: 57-66

8 Miyamoto S, Mori K, Ihara H, et al. Autonomous decentralized control and its application to the rapid transit system. Comput Ind, 1984, 5: 115-124

9 Mori K. Autonomous decentralized system technologies and their application to train transport operation system. In: Winter V L, Bhattacharya S, eds. High Integrity Software. Heidelberg: Springer, 2001. 89-111

10 Kravitz E A. Hormonal control of behavior: amines and the biasing of behavioral output in lobsters. Science, 1988, 241: $1175-1181$

11 Brooks R A. Integrated systems based on behaviors. SIGART Bull, 1991, 2: 46-50

12 Avila-Garcia O, Canamero L. Using hormonal feedback to modulate action selection in a competitive scenario. In: Proceedings of the 8th International Conference on Simulation of Adaptive Behavior. Los Angeles, 2004. 243-252

13 Avila-Garcia O, Canamero L. Hormonal modulation of perception in motivation-based action selection architectures. In: Proceedings of the AISB'05 Symposium. Hatfield, 2005. 9-16

14 Shen W M, Chuong C M, Will P. Digital hormone models for self-organization. In: Proceedings of the 8th International Conference on Artificial Life. Sydney, 2002. 116-120 
15 Shen W M, Chuong C M, Will P. Simulating self-organization for multi-robot systems. In: 2002 IEEE/RSJ International Conference on Intelligent Robots and System. Lausanne, 2002. 2776-2781

16 Shen W M. Self-organization through digital hormones. IEEE Intell Syst, 2003, 18: 81-83

17 Shen W M, Will P, Galstyan A, et al. Hormone-inspired self-organization and distributed control of robotic swarms. Auton Robot, 2004, 17: 93-105

18 Jiang $\mathrm{T}$ X, Widelitz R B, Shen W M, et al. Integument pattern formation involves genetic and epigenetic controls: Feather arrays simulated by digital hormone models. Int J Dev Biol, 2004, 48: 117-135

19 Bayindir L, Sahin E. A review of studies in swarm robotics. Turk J Elec Eng \& Comp Sci, 2007, 15: 115-147

20 Shen W M, Salemi B, Will P. Hormones for self-reconfigurable robots. In: Proceedings of the 6th International Conference on Intelligent Autonomous Systems. Venice, 2000. 918-925

21 Shen W M, Lu Y M, Will P. Hormone-based control for self-reconfigurable robots. In: Proceedings of the 4th International Conference on Autonomous Agents. Barcelona, 2000. 1-8

22 Salemi B, Shen W M, Will P. Hormone-controlled metamorphic robots. In: Proceedings of 2001 IEEE International Conference on Robotics and Automation. Seoul, 2001. 4194-4199

23 Shen W M, Salemi B, Will P. Hormone-inspired adaptive communication and distributed control for CONRO selfreconfigurable robots. IEEE Trans Robot Automat, 2002, 18: 700-712

24 Krivokon M, Will P, Shen W M. Hormone-inspired distributed control of self-reconfiguration. In: Proceedings of 2005 IEEE International Conference on Networking, Sensing and Control. Tucson, 2005. 514-519

25 Mendao M. A neuro-endocrine control architecture applied to mobile robotics. Dissertation for the Doctoral Degree. Canterbury: University of Kent, 2007. 1-49

26 Walker J, Wilson M. A performance sensitive hormone-inspired system for task distribution amongst evolving robots. In: Proceedings of 2008 IEEE/RSJ International Conference on Intelligent Robots and Systems. Nice, 2008. 1293-1298

27 Sugano S, Ogata T. Emergence of mind in robots for human interface - research methodology and robot model. In: Proceedings of 1996 IEEE International Conference on Robotics and Automation. Minneapolis, 1996. 1191-1198

28 Ogata T, Sugano S. Emotional communication between humans and the autonomous robot which has the emotion model. In: Proceedings of 1999 IEEE International Conference on Robotics and Automation. Detroit, 1999. 3177-3182

29 Huang G R. Research on artificial endocrine models and its applications. Dissertation for the Doctoral Degree. Hefei: University of Science \& Technology of China, 2003. 59-78

30 Greensted A J, Tyrrell A M. Fault tolerance via endocrinologic based communication for multiprocessor systems. In: Proceedings of the 5th International Conference on Evolvable Systems: from Biology to Hardware. Trondheim, 2003. $24-34$

31 Greensted A J, Tyrrell A M. An endocrinologic-inspired hardware implementation of a multicellular system. In: Proceedings of 2004 NASA/DOD Conference on Evolution Hardware. Seattle, 2004. 245-252

32 Greensted A J, Tyrrell A M. Implementation results for a fault-tolerant multicellular architecture inspired by endocrine communication. In: Proceedings of 2005 NASA/DOD Conference on Evolvable Hardware. Washington DC, 2005. 253261

33 Liu B, Han H, Ding Y S. A decoupling control based on the bi-regulation principle of growth hormone. In: Proceedings of 2005 ICSC Congress on Computational Intelligence: Methods \& Application. Istanbul, 2005. 1-4

34 Liu B, Zhang Z W, Ding Y S. Decoupling control based on bi-directional regulation principle of growth hormone (in Chinese). J Southeast Univ (Nat Sci Ed), 2006, 36: 5-8

35 Liu B. Bio-network-based intelligent control systems and their applications. Dissertation for the Doctoral Degree. Shanghai: Donghua University, 2006. 35-68

36 Balasubramaniam S, Botvich D, Donnelly W, et al. A biologically inspired policy based management system for survivability in autonomic networks. In: Proceedings of the 4th International Conference on Broadband Communications, Networks and System. Raleigh, 2007. 160-168

37 Brinkschulte U, Pacher M, Von Renteln A. Towards an artificial hormone system for self-organizing real-time task allocation. In: Proceedings of the 5th IFIP Workshop on Software Technologies for Future Embedded and Ubiquitous Systems. Santorini Island, 2007. 339-347

38 Brinkschulte U, Pacher M, Von Renteln A. An artificial hormone system for self-organizing real-time task allocation in organic middleware. In: Wurtz R P, ed. Organic Computing. Understanding Complex Systems. Heidelberg: Springer, 
2008. 261-283

39 Von Renteln A, Brinkschulte U, Weiss M. Examinating task distribution by an artificial hormone system based middleware. In: the 11th IEEE Symposium on Object Oriented Real-Time Distributed Computing. Orlando, 2008. 119-123

40 Xu Q Z, Wang L. Recent advances in artificial endocrine system. J Zhejiang Univ Sci C Comput Electr, 2011, 12: 171-183

41 Yang G. Physiology \& Path Physiology (in Chinese). Tianjin: Tianjin Scientific \& Technical Publishers, 1996. 1-76

42 Felig P, Frohman L A. Endocrinology and Metabolism. 4th ed. Ohio: The McGraw-Hill Companies, 2001. 3-18

43 Liao E Y, Mou Z H. Endocrinology (in Chinese). 2nd ed. Beijing: People's Medical Publishing House, 2007. 1-67

44 Gardner M. The fantastic combinations of John Conway's new solitaire game life. Sci Am, 1970, 223: 120-123

\title{
Lattice-based artificial endocrine system model and its application in robotic swarms
}

\author{
WANG Lei ${ }^{1} \&$ XU QingZheng ${ }^{1,2 *}$ \\ 1 School of Computer Science and Engineering, Xi'an University of Technology, Xi'an 710048, China; \\ 2 Xi'an Communication Institute, Xi'an 710106, China \\ *E-mail: xuqingzheng@hotmail.com
}

\begin{abstract}
To solve the problem of controlling robot swarms in a distributed manner, we propose a novel latticebased artificial endocrine system (LAES) model, inspired by modern endocrinology theory. Based on a latticed environment, relying on cell intelligentization, connected by cumulative hormones, and directed by target cells, the LAES model can finally adapt to the continuous volatility of the external environment and maintain the relevant stability of the internal dynamics of the system, thus exhibiting the self-organizing and self-repairing features of the biological endocrine system. Experiments show that the LAES model enables a robotic swarm to search an unfamiliar space and seize multiple targets automatically without using unique global identifiers or a centralized control strategy for the individual robots.
\end{abstract}

Keywords artificial endocrine system, robotic swarms, endocrine cell, hormone, self organization, self repair, searching and seizing 